\title{
MOVIMIENTO SINISTRAL EN EL CRETACICO INFERIOR DE LA ZONA DE FALLA ATACAMA AL NORTE DE PAPOSO (24ㅇ), CHILE
}

\section{MIGUEL HERVE A.}

Servicio Nacional de Geologla y Minería, Casilla 10465, Santiago, Chile

\begin{abstract}
RESUMEN
La zona de Falla Atacama, que se extiende por unos $1.000 \mathrm{~km}$ en la Cordillera de la Costa del norte de Chile, ha tenido, a lo largo de su actividad meso-cenozoica, episodios de transcurrencia que han generado extensas franjas de rocas miloníticas. Hasta este momento, no habia sido posible determinar el sentido de este movimiento horizontal relativo.

El estudio geológico de las rocas plutónicas que forman el batolito costero, en el área al norte de Paposo, ha permitido identificar un plutón granodiorítico que está desplazado $34 \mathrm{~km}$, en el sentido sinistral, a lo largo del Sistema de Falla Izcuña, que está incluido en la Zona de Falla Atacama. Se trata del primer antecedente de terreno que permite establecer el sentido y la magnitud del movimiento en la Zona de Falla Atacama. Este movimiento transcurrió en el Cretácico Inferior entre los $144 \mathrm{Ma}$ (edad del plutón desplazado) y los $131 \mathrm{Ma}$ (edad de un granito post-milonitización). Una milonita tue datada en $139 \mathrm{Ma}$, confirmando así la edad supuesta para su formación.

La Zona de Falla Atacama se dispone a lo largo de un arco magmático mesozoico, de carácter calcoalcalino, relacionado con una zona de subducción. Su actividad transcurrente tuvo lugar cerca de la culminación de la evolución de la actividad magmática del arco. La presencia de sistemas de fallas transcurrentes, paralelos al sistema arco-fosa, delatan subducción oblicua. Se propone aqui, que en el borde continental de este sector del norte de Chile, durante el Mesozoico, se produjo esta situación de convergencia oblicua, con una fuerte componente NW-SE del movimiento de la placa oceánica subductada. De esta forma, se genera un sistema de fallas sinistrales similar al descrito.
\end{abstract}

Palabras claves: Falla Atacama, Movimiento sinistral, Datación K-Ar, Convergencia oblicua, Norte de Chile.

\section{ABSTRACT}

The Atacama Fault Zone, extending some $1,000 \mathrm{~km}$ along the Coastal Range of northern Chile, includes in its MesoCenozoic evolution, mylonite forming strike-slip faulting episodes. Until now, no evidence regarding the sense of the horizontal relative displacement had been found.

The geological study of the plutonic rocks belonging to a Mesozoic coastal batholith north of Paposo, has allowed the identification of a granodioritic pluton that has been displaced $34 \mathrm{~km}$ by sinistral movement along the Izcuña Fault System (included in the Atacama Fault System). This is the first field evidence of the sense of horizontal movement in the Atacama Fault Zone. The sinistral movement occurred in the lowermost Cretaceous, between $144 \mathrm{Ma}$ (age of the displaced pluton) and $131 \mathrm{Ma}$ (age of a post-mylonitic granite). The age of $139 \mathrm{Ma}$, obtained from a mylonite, is concordant with the time span here proposed.

The Atacama Fault Zone lies within a Mesozoic calcalkaline subduction related magmatic arc, and its strike-slip activity occurred close to the culmination of the magmatic evolution of the arc. Strike-slip faults paralleling the arc-trench system are thought to be generated by oblique subduction. Such a system, with a strong NW to SE component of movement is proposed here for the continental margin of this part of northern Chile, in Early Cretaceous time, in order to produce the sinistral strike-slip fault systems described.

Key words: Alacama fault, Sinistral movement, K-Ar dating, Oblique convergence, Northern Chile.

\section{INTRODUCCION}

La Zona de Falla Atacama constituye una franja de dirección general norte-sur, que ha sido recono- 
cida a lo largo de, por lo menos, $1.000 \mathrm{~km}$ en la Cordillera de la Costa del Norte Grande de Chile. Se ha sostenido que es una estructura regional de tipo transcurrente (St. Amand et al., 1960; Arabasz, 1971; Naranjo et al., 1984) aunque, a la fecha, no ha sido posible precisar la magnitud ni el sentido del movimiento horizontal relativo para ella.

La Zona de Falla Atacama fue subdividida, longitudinalmente, por Arabasz (1971) y, al norte de Paposo, la falla principal se denominó Falla Salar del Carmen (Falla Paposo de Hervé, 1987) (Fig. 1). Unos $10 \mathrm{~km}$ al oeste de esta falla y con una orientación paralela, Arabasz (1971) definió la Falla Izcuña. Esta última tiene aproximadamente 60 $\mathrm{km}$ de largo y su límite sur se ubica en el acantilado costero, algunos kilometros al norte de Paposo. Esta falla, junto a otras asociadas a ella, se denomina aqui Sistema de Falla Izcuña. Sobre ésta se han hecho las observaciones geológicas que se presentan en este artículo. La zona de estudio queda limitada al este por la traza de la Falla Paposo-Salar del Carmen y al oeste por el acantilado costero. Alcanza desde Paposo por el sur hasta las cercanías del camino Antóagasta-Caleta El Cobre por el norte.

El levantamiento geológico de la Cordillera de la Costa al norte de Paposo (Proyecto Aguas Blancas, escala 1:250.000, Carta Geológica de Chile, del Servicio Nacional de Geología y Minería) ha permitido distinguir diferentes plutones, en el batolito costero, que constituyen la gran mayoría de los afloramientos del área, y generar la nomenclatura que se utiliza en este texto. Se ha verificado, además, el efecto que ha tenido la actividad del sistema de falla ya mencionado, en estas rocas. Así es como se ha llegado a establecer que un plutón granodiorítico se encuentra seccionado y desplazado sinistralmente por el Sistema de Falla Izcuña. Se presenta aquí el análisis de la magnitud y sentido del movimiento horizontal relativo, junto al análisis de dataciones radiométricas K-Ar en plutones y rocas de falla, lo que permite estimar la probable edad del movimiento horizontal.

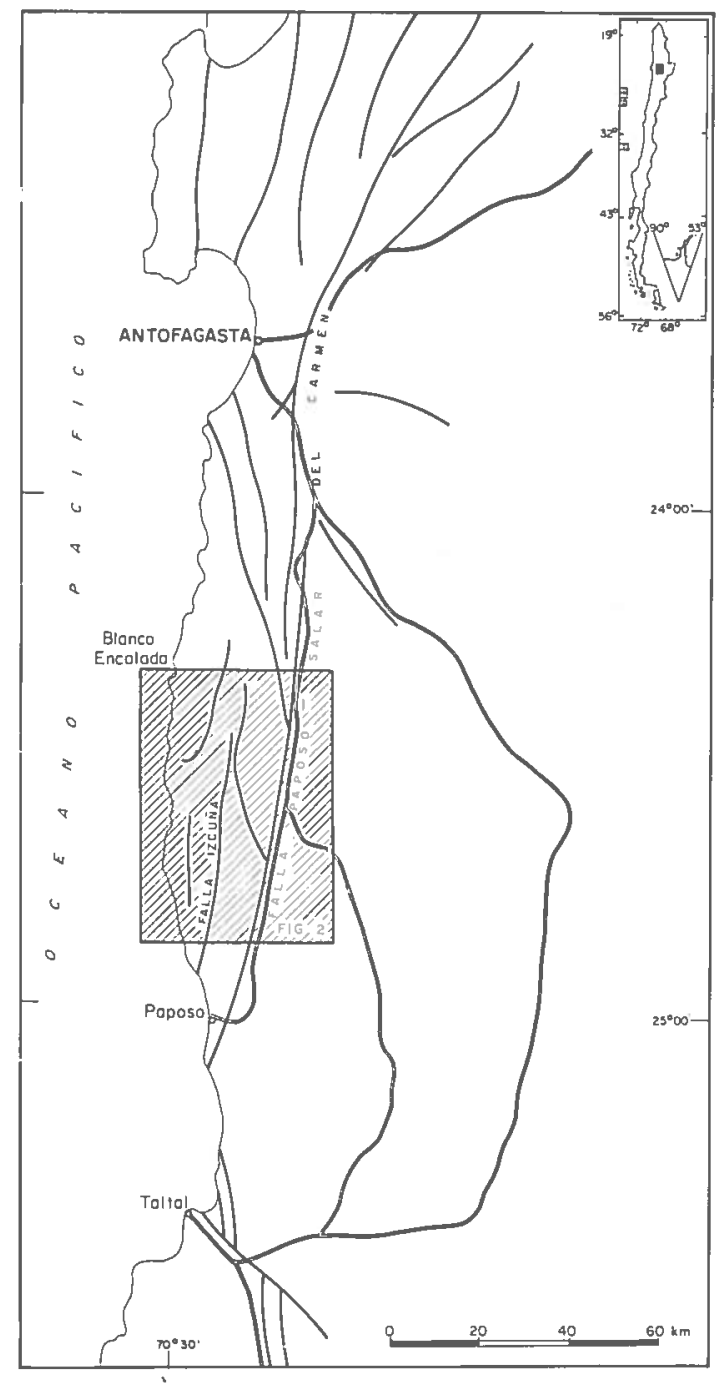

FIG. 1. Ubicación del área estudiada, con las principales estructuras del Sistema de Falla Atacama e indicación del área representada en la figura 2.

\section{ANTECEDENTES GEOLOGICOS GENERALES}

En la zona de este estudio, predominan las rocas plutónicas de edad mesozoica, con un amplio rango de composición mineralógica. En el sector noroccidental de Blanco Encalada, afloran pórfidos andesíticos, relacionados genética y temporalmente con la Formación La Negra, del Jurásico, definida por García (1967).

Las rocas plutónicas más abundantes son del ti- 
po gabroides y se han agrupado en una unidad denominada Gabros Cerro Paranal, compuesta por gabros, noritas y dioritas, con sus variedades monzoníticas y cuarcíferas y edades de cristalización jurásicas. Los gabroides se encuentran intruidos por rocas mas leucocráticas, tales como granodioritas y granitos, de dimensiones más reducidas y edades cretácicas inferiores, que han sido agrupadas en diversas unidades plutónicas. El plutón granodiorítico que se analiza aquí, pertenece a la unidad Granitoides Cerro Ventarrones.

Todas las rocas ya mencionadas se encuentran surcadas por fallas pertenecientes a la Zona de Falla Atacama. La dirección preferencial es norte-sur aunque, localmente, hay fallas o segmentos de ellas con rumbos oblicuos respecto a la falla principal. Muchas de estas fallas presentan una marcada expresión topográfica, debido a que se han reactivado en el Terciario Superior con un carácter normal (Hervé, 1987). Generalmente, a lo largo de ellas se alinean quebradas parcialmente rellenadas con material coluvial y aluvial. Se han observado rocas de falla, del tipo milonitas, solamente en aquellos afloramientos de rocas inmediatamente adyacentes a las estructuras, como, por ejemplo, en Quebrada Remiendos al interior de Blanco Encalada (Fig. 2). Estas milonitas están intruidas por un sienogranito sin deformación interna.

El plutón, en el cual se verificaron las evidencias de un desplazamiento horizontal relativo a to largo de las fallas del Sistema Izcuña, es una granodiorita de biotita. Exhibe texturas hipidiomorfas granulares, en parte porfíricas, con grano grueso a muy grueso y fenocristales de ortoclasa de hasta $4 \mathrm{~cm}$ de longitud. Este carácter iuertemente porfíri$\mathrm{co}$, aunque presente sólo ocasionalmente en la roca, es importante para identificar los diferentes segmentos de este plutón, como integrantes de un solo cuerpo plutónico.

En la granodiorita no se ha reconocido la presencia de foliación ni bandeamiento. Hay que hacer notar la ausencia de afloramientos adecuados en las inmediaciones de las trazas de las fallas, los que, en general, se presentan intensamente meteorizados o cubiertos.

Los minerales más abundantes en este plutón son el cuarzo y la plagioclasa. El cuarzo (30-40\%) se presenta en cristales intersticiales con extinción ondulosa moderada a nula, sin evidencias de deformación intensa. La plagioclasa $(30-40 \%)$, del tipo andesina, se presenta con zonación alterna, en cristales subhedrales menores que $0,5 \mathrm{~cm}$ y escasamente sericitizados. El feldespato potásico es ortoclasa, con macla de Carlsbad cuando se desarrolla en cristales de hasta $4 \mathrm{~cm}$. El mineral ferromagnesiano más abundante es la biotita (3-5\%), en cristales subhedrales levemente curvados en sectores. La hornblenda es escasa $(<1 \%)$, aparece en cristales tabulares, sin alteración, algunos con núcleos de clinopiroxeno. Los minerales accesorios reconocidos son apatita, circón, esfeno y opacos.

Se han obtenido edades K-Ar en biotita, de 144 y $143 \mathrm{Ma}$, en diferentes segmentos del plutón granodiorítico (Tabla 1; Fig. 2), que se interpretan como cercanas a la cristalización. En la unidad Gabros Cerro Paranal, que constituye la roca encajadora, se han obtenido edades K-Ar de $144 \mathrm{Ma}$, en las cercanías de la granodiorita, y de $155 \mathrm{Ma}$ o más antiguas, en sectores más alejados (Fig. 2). Las edades más antiguas se interpretan como edades mínimas de cristalización de la unidad de gabros, mientras que las cercanas a los $144 \mathrm{Ma}$, se interpretan como rejuvenecidas por el evento térmico que acompañó al proceso de intrusión de la granodiorita.

\section{EVIDENCIAS DE DESPLAZAMIENTO SINISTRAL Y EDAD DEL MOVIMIENTO}

En el mapa geológico esquemático de la figura 2 , se destaca, sobre la base de la posición actual del plutón granodiorítico, el desplazamiento horizontal relativo de carácter sinistral, que ha afectado a las estructuras del Sistema de Falla Izcuña. Los extremos del plutón se encuentran, actualmente, a una distancia de $34 \mathrm{~km}$, en el sentido general de las fallas que lo desplazan. A lo largo de la Falla
Izcuña propiamente tal, el desplazamiento sinistral es de unos $15 \mathrm{~km}, y$ es el de mayor magnitud observado en el sistema. Los $19 \mathrm{~km}$ restantes se distribuyen en desplazamientos inferiores a $6 \mathrm{~km}$, a lo largo de las otras cuatro fallas del sistema.

La edad máxima posible, para el movimiento horizontal relativo, está dada por la edad de la roca más joven afectada por el movimiento, que, en es- 


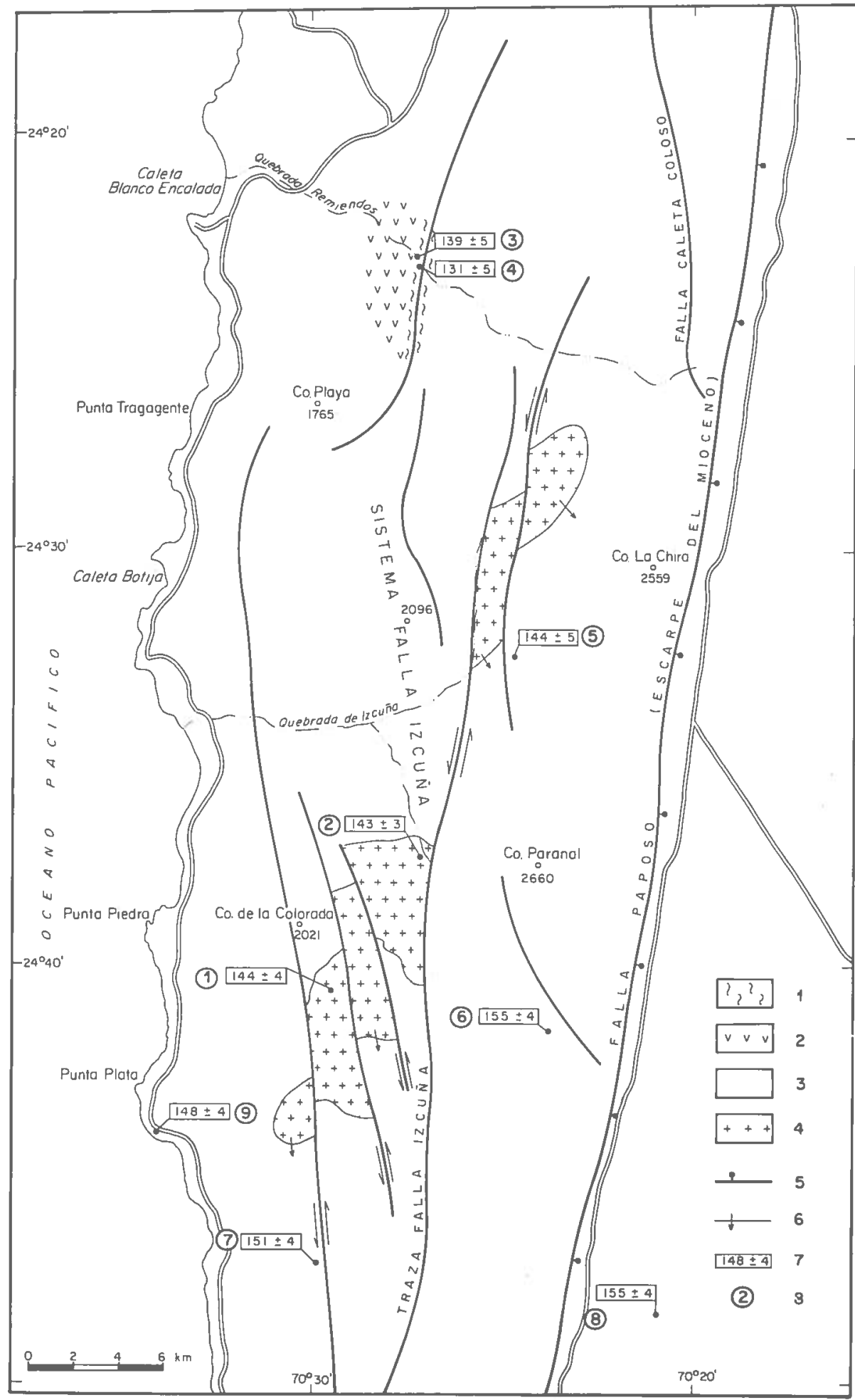

Fig. 2. Bosquejo geológico del área estudiada, con indicación de la ubicación y edad de las muestras datadas por el método K-Ar. 1. Milonitas; 2. Andesitas porfíricas; 3. Plutones indiferenciados; 4 . Granodiorita porfírica; 5 . Falla; 6 . Flecha indica el plutón intruido; 7. Edad K-Ar en biotita (Ma); 8 . Número de datación en Tabla 1. 
TABLA 1. RESULTADOS ANALITICOS DE LAS DATACIONES K-AR

\begin{tabular}{|c|c|c|c|c|c|c|}
\hline Muestra & $\begin{array}{l}\text { Tipo } \\
\text { Litológico }\end{array}$ & Material & $\% \mathrm{~K}$ & $\begin{array}{l}{ }^{40} \mathrm{Ar} \mathrm{rad} . \\
\mathrm{nl} / \mathrm{g} \text { (STP) }\end{array}$ & $\begin{array}{l}\text { \%Ar } \\
\text { Alm. }\end{array}$ & $\begin{array}{l}\text { Edad y Error } \\
\qquad \mathrm{Ma}(2 \sigma)\end{array}$ \\
\hline \multicolumn{7}{|l|}{$\begin{array}{l}\text { Granodlorita } \\
\text { desplazada }\end{array}$} \\
\hline 1 HАВ-505 & Granodiorita & Biotita & 6,658 & 38,739 & 15 & $144 \pm 4$ \\
\hline 2 HAB-525 & Granodiorita & Biotita & 7,376 & 42,567 & 11 & $143 \pm 3$ \\
\hline \multicolumn{7}{|l|}{ Milonita } \\
\hline 3 HAB-648A & Milonita & Roca total & 1,769 & 9,911 & 33 & $139 \pm 5$ \\
\hline \multicolumn{7}{|c|}{$\begin{array}{l}\text { Granitos post } \\
\text { milonita }\end{array}$} \\
\hline 4 HAB-689 & Sienogranito & Biotita & 2,432 & 12,851 & 21 & $131 \pm 5$ \\
\hline \multicolumn{7}{|c|}{ Gabros y dioritas } \\
\hline $\begin{array}{l}\text { Cerro Paran } \\
5 \mathrm{HAB}-412\end{array}$ & Gabro & Biotita & 3,736 & 21,785 & 26 & $144 \pm 5$ \\
\hline 6 HAB-518 & Gabro & Biotita & 5,944 & 37,339 & 14 & $155 \pm 4$ \\
\hline 7 HAB-613 & Diorita & Biotita & 7,055 & 43,067 & 11 & $151 \pm 4$ \\
\hline 8 HAB-586 & Monzogabro & Biotita & 3,219 & 20,222 & 21 & $155 \pm 4$ \\
\hline 9 HAB-10 & Gabro & Biolita & 5,409 & 32,373 & 12 & $148 \pm 4$ \\
\hline
\end{tabular}

Tocias las edades se han obtenido en el Laboratorio de Geocronologla K-Ar del Servicio Nacional de Geologia y Mineria. Constantes utilizadas según Steiger y Jäger, 1977.

te caso, corresponde a la edad cretácica inferior (144 Ma) obtenida en la granodiorita. Asimismo, la edad mínima para el movimiento está definida por la roca más antigua que no esté afectada por las fallas, en este caso, la edad de 131 Ma en biotita, obtenida en un sienogranito, que intruye a las milonitas en el sector de Quebrada Remiendos (Tabla 1; Fig. 2).

La datación en roca total de una milonita andesítica, en el mismo lugar, indicó $139 \mathrm{Ma}$, edad que se interpreta como rejuvenecida por eventos térmicos relacionados con procesos de milonitización, por lo que indicaría la edad de la actividad transcurrente de la falla.

Se define, de este modo, un intervalo entre los 131 y $144 \mathrm{Ma}$, en el que debe haber ocurrido el movimiento transcurrente del sistema de fallas y la milonitización de las rocas. La edad concordante de la milonita estaría, así, confirmando esta época cretácica inferior para el desplazamiento horizontal sinistral.

La milonita de Quebrada Remiendos, con la que se define la cronología de estas rocas, no está en la continuación directa hacia el norte de ninguna de las fallas del Sistema Izcuña (Fig. 2), pero, debido a la similitud geológica y a la cercanía entre ellas, se les supone una evolución tectónica comparable.

La cronología aqui descrita coincide con las edades del Cretácico Inferior, obtenidas para la milonitización en otro segmento de la Zona de Falla Atacama, situado unos $300 \mathrm{~km}$ más al sur (Naranjo et al., 1984).

\section{CONCLUSIONES}

La distribución de los afloramientos de un plutón granodiorítico desplazado por el Sistema de Falla Izcuña, se explica por la acción de movimientos transcurrentes de carácter sinistral. Este tipo de movimiento se identifica aquí, por primera vez, en fallas pertenecientes a la Zona de Falla Atacama.
El movimiento transcurrente habría ocurrido en el intervalo entre los $144 \mathrm{Ma}$ y $131 \mathrm{Ma}$, en el Cretácico Inferior bajo, sobre la base del análisis de dataciones radiométricas $\mathrm{K}$-Ar.

El fallamiento transcurrente sinistral tuvo lugar en un momento en el cual estaba culminando la 
evolución de un arco magmático del Mesozoico en el borde continental de Chile a esta latitud (Hervé et al., 1985). Los plutones graníticos que intruyen a las milonitas son los plutones más jóvenes emplazados al oeste de la traza principal de la Falla Atacama, ya que, los del Cretácico Superior lo hacen más al este. El magmatismo del arco se relaciona genéticamente con un régimen de subducción de una placa oceánica bajo una continental. Si se supone que el movimiento transcurrente sinistral tuvo un carácter regional, es preciso que la subducción haya sido oblicua con respecto al margen continental, con una componente importante del noroeste.

En la actualidad, no es posible conocer la posición de otros elementos morfológicos del sistema arco-fosa mesozoico, tales como el eje del arco magmático, el frente volcánico y la plataforma de ante-arco, entre otros, debido principalmente a la ausencia de rocas estratificadas que caracterizan a estos elementos. Al mismo tiempo, este hecho indica un nivel actual de erosión profundo de estas rocas. Por otro lado, es posible que, debido a un régimen con erosión tectónica (Uyeda, 1983), el arco magmático se haya erodado parcialmente.

\section{AGRADECIMIENTOS}

Este trabajo forma parte del Proyecto No. 202 del Programa Internacional de Correlación Geoló- gica denominado Megafallas de Sudamérica.

\section{REFERENCIAS}

ARABASZ, W.J. 1971. Geological and Geophysical studies of the Atacama Fault Zone in Northern Chile. Ph.D. Thesis. California Institute of Technology, 264 p. Pasadena, U.S.A.

GARCIA, F. 1967. Geología del Norte Grande de Chile. In Simposium sobre el Geosinclinal Andino, No. 3, Sociedad Geológica de Chile, 138 p. Santiago, Chile.

HERVE, M. 1987. Movimiento normal de la Falla Paposo, Zona de Falla Atacama, en el Mioceno, Chile. Revista Geológica de Chile, No. 31, p. 31-36. Este volumen.

HERVE, M.; MARINOVIC, N.; MPODOZIS, C.; PEREZ DE ARCE, C. 1985. Geocronología K-Ar de la Cordillera de la Costa entre los $24^{\circ}$ y $25^{\circ} \mathrm{S}$ : Antecedentes preliminares. In Congreso Geológico Chileno, No. 4, Resúme- nes, p. 158. Antofagasta, Chile.

NARANJo, J.A.; heRVE, F.; PRIETO, X.; munizaga, F. 1984. Actividad cretácica de la Falla Atacama al este de Chañaral: Milonitización y plutonismo. Comunicaciones, No. 34, p. 57-66.

St. AMAND, P.; ALLEN, C.R. 1960. Strike-slip faulting in Northern Chile. Geological Society of America, Bulletin [Abstracts], Vol. 71, p. 1965.

STEIGER, R.H.; JÄGER, E. 1977. Subcommission in Geochronology: Conventions on the use of decay constants in Geo and Cosmochronology. Earth and Planetary Science Letters, Vol. 36, p. 359-362.

UYEDA, S. 1983. Comparative subductology. Episodes, Vol. 2, p. 19-24. 